Ebisu Ebisu

Études japonaises

49 | printemps-été 2013

De chose en fait : la question du milieu

Shinobu CHUJO, avec son équipe (Tetsuro NEGISHI, Atsushi ODE, Nobutaka SHINONAGA), introduction de Jacques HOURIEZ, Chronologie de Paul Claudel au Japon

Paris, Éditions Honoré Champion, coll. « Poétiques et esthétiques $\mathrm{Xx}^{\mathrm{e}}-\mathrm{xxI} \mathrm{e}^{\mathrm{e}}$ siècles ", 2012, 665 p.

Christophe Marquet

\title{
OpenEdition
}

Journals

Édition électronique

URL : http://journals.openedition.org/ebisu/840

DOI : $10.4000 /$ ebisu. 840

ISSN : 2189-1893

Éditeur

Institut français de recherche sur le Japon à la Maison franco-japonaise (UMIFRE 19 MEAE-CNRS)

Édition imprimée

Date de publication : 1 avril 2013

Pagination : 181-186

ISSN : 1340-3656

Référence électronique

Christophe Marquet, « Shinobu Chuso, avec son équipe (Tetsuro NEGISHI, Atsushi oDE, Nobutaka SHinonagA), introduction de Jacques houriez, Chronologie de Paul Claudel au Japon », Ebisu [En ligne], 49। printemps-été 2013, mis en ligne le 25 mars 2014, consulté le 22 septembre 2020. URL : http:// journals.openedition.org/ebisu/840 ; DOI : https://doi.org/10.4000/ebisu.840

Ce document a été généré automatiquement le 22 septembre 2020.

(c) Institut français de recherche sur le Japon à la Maison franco-japonaise 


\section{Shinobu $\mathrm{CHUJO}_{\mathrm{H}}$, avec son équipe (Tetsuro NEGISHI, Atsushi ODE,} Nobutaka SHINONAGA), introduction de Jacques Houriez, Chronologie de Paul Claudel au Japon

Paris, Éditions Honoré Champion, coll. «Poétiques et esthétiques $\mathrm{XX}^{\mathrm{e}}-\mathrm{XXI}^{\mathrm{e}}$ siècles », 2012, 665 p.

\section{Christophe Marquet}

\section{RÉFÉRENCE}

Shinobu CHUJO, avec son équipe (Tetsuro NEGISHI, Atsushi ODE, Nobutaka SHINONAGA), introduction de Jacques HOURIEZ, Chronologie de Paul Claudel au Japon, Paris, Éditions Honoré Champion, coll. "Poétiques et esthétiques $\mathrm{XX}^{\mathrm{e}}-\mathrm{XXI}^{\mathrm{e}}$ siècles », 2012, 665 p. Publié avec le concours de la Shibusawa Eiichi Memorial Foundation.

1 Au moment où la Maison franco-japonaise s'apprête à fêter en 2014 son quatre-vingtdixième anniversaire, cet ouvrage arrive à point nommé. Il permet de replacer cette commémoration dans le contexte des années 1920 au Japon et de préciser le rôle joué par Paul Claudel - ambassadeur de France au Japon de 1921 à 1927 -, dans le renforcement des relations entre les deux pays au lendemain de la Première Guerre mondiale.

2 Cette somme est le fruit du travail de toute une équipe, dirigée par le Pr. Chūjō Shinobu. Il en existe une version japonaise, publiée en 2010 aux éditions Kuresu à Tokyo ${ }^{1}$. La version française est augmentée d'une longue et instructive introduction rédigée par Jacques Houriez (p.9-34), collaborateur de l'édition du théâtre de Claudel 
dans la «Bibliothèque de la Pléiade » et qui est aussi l'auteur dans la même collection des éditions Champion de Paul Claudel, ou les tribulations d'un poète ambassadeur. Chine, Japon, Paris (2012).

3 Chūjō Shinobu, professeur honoraire à l'université Aoyama gakuin à Tokyo, est lui aussi un spécialiste du théâtre de Claudel et notamment de ses liens avec le théâtre japonais. Il a également publié une traduction japonaise du recueil Contact et circonstances de Claudel et fut le co-organisateur du grand colloque Claudel et le Japon ${ }^{2}$ et de l'exposition commémorative organisés en 2005 pour le cinquantième anniversaire de la mort de l'écrivain ${ }^{3}$. Nous renvoyons également à son intéressant article «Paul Claudel et la fondation de la Maison franco-japonaise ", publié dans le n ${ }^{\circ} 26$ de la revue Ebisu en 2001.

4 Le présent ouvrage a surtout une grande valeur documentaire, puisqu'il utilise de nombreuses sources de première main (dossiers personnels et publics des archives françaises et japonaises, dépêches et correspondances diplomatiques) sur la présence de Claudel au Japon, sources qui viennent s'ajouter aux informations tirées de la presse japonaise de l'époque ou du Journal de Claudel, et qui sont classées de manière chronologique : du 27 mai au 21 juin 1898 pour un premier voyage de découverte du Japon alors que Claudel est en poste au consulat de Shanghai, puis de novembre 1921 à février 1927, lors de son séjour comme ambassadeur.

5 L'ouvrage est accompagné d'une bibliographie et de deux index des noms de personnes et des lieux, institutions et événements. Il sera donc un outil utile non seulement aux spécialistes de Claudel, mais aussi aux chercheurs qui travaillent sur l'histoire des relations franco-japonaises à cette période. Il comporte vingt-huit rares documents photographiques sur Claudel au Japon - dont une photographie du premier bâtiment de la Maison franco-japonaise -, mais on regrettera l'assez piètre qualité de leur reproduction (l'édition japonaise qui reproduit les mêmes documents est de bien meilleure qualité sur ce point).

6 À côté des données à caractère parfois anecdotique sur l'emploi du temps officiel de Claudel ambassadeur et sur ses multiples déplacements, on peut y lire de précieuses informations de nature politique, économique ou militaire, qui éclairent la connaissance de cette période.

7 En ce qui concerne la création de la Maison franco-japonaise, on apprend toute une série de faits que nous résumerons ici $^{4}$. Cette institution fut initialement appelée "Maison de France » dans les premiers écrits, ce qui suggère que l'on passa de l'idée initiale d'en faire un lieu exclusivement consacré à la promotion de la langue et de la culture françaises, à une structure d'échanges culturels et académiques entre les deux pays.

8 La première mention publique du projet date du 17 décembre 1921, à l'occasion d'un dîner organisé par la Société franco-japonaise (créée en 1909) à la Maison des Nobles (p.75). C'est un mois à peine après l'arrivée de Claudel au Japon, ce qui indique combien ce projet était prioritaire dans les missions confiées par le gouvernement français au nouvel ambassadeur. De fait, les Instructions remises par le Président de la République Millerand au diplomate à la veille de son départ comportaient deux priorités: le développement des exportations françaises (dans les secteurs de l'armement et de l'aéronautique notamment) et la diffusion de la langue et de la culture 
françaises, dans le but de contrebalancer les influences anglaises et allemandes au lendemain de la victoire ${ }^{5}$.

9 On apprend que le dessein initial prévoyait que la France consacre 300000 francs par an pour l'envoi au Japon d'un spécialiste et de quatre ou cinq étudiants français, tandis que le Japon s'occuperait des frais de logement, des salles de travail et de la bibliothèque (p. 76-77). Claudel espérait récolter par ailleurs 300000 yens au Japon (p. 84) par souscription et s'assurer l'aide du Gouvernement général de l'Indochine. Le lien avec la colonie française se maintiendra dans les années 1930 et plusieurs membres de l'École française d'Extrême-Orient (Paul Demiéville, Élie Auboin, Émile Gaspardone), installée à Saigon et à Hanoi depuis le début du siècle, deviendront les premiers pensionnaires de la Maison. Le comité fondateur comptait aussi sur une subvention de 60000 yens du gouvernement japonais ${ }^{6}$ (p. 173).

Une correspondance du 16 janvier 1922 (p. 85) nous fait savoir que pour la direction de la future Maison, Claudel avait d'abord eu une préférence pour l'historien de l'art médiéval Henri Focillon (1881-1943), alors directeur du musée des beaux-arts de Lyon et qui, rappelons-le, fut l'auteur en 1914 d'un ouvrage sur Hokusai et en 1921 d'une étude sur l'art bouddhique. Le premier directeur proposé par le gouvernement français sera finalement le grand indianiste Sylvain Lévi (1863-1935), professeur au Collège de France et personnalité déjà connue au Japon (p. 175), pour avoir eu des élèves japonais dans ses cours à l'École pratique des hautes études et pour y avoir séjourné dès 1898 . Claudel avait néanmoins manifesté dans un premier temps sa réserve à propos de sa "vision de l'histoire » (p. 85) et s'inquiéta à plusieurs reprises de l'origine juive de ce dernier (p. 265, p. 368) qui était le président de l'Alliance israélite universelle.

Dans une autre correspondance, adressée à Jean Giraudoux (qui travaillait alors dans le Service de l'œuvre française à l'étranger au ministère des Affaires étrangères) le 22 février 1922 (p. 98), on comprend bien le projet stratégique de Claudel à travers cette maison, qui est de resserrer les liens entre la France et le Japon, «parce que le Japon dominera l'Asie » et qu'il faut lutter contre les influences américaine et allemande qui s'y exercent. La littérature et la médecine sont considérées par Claudel comme deux domaines d'influence prioritaires.

Le projet de cette Maison franco-japonaise se veut aussi comme un pendant à la Maison du Japon à la Cité universitaire internationale - dont la création est établie dès juin 1921 -, où elle sera construite entre 1927 et 1929 grâce à l'aide financière de l'homme d'affaires Satsuma Jirohachi (p. 144).

Les difficultés sont néanmoins nombreuses pour collecter les fonds destinés à la Maison franco-japonaise, à cause de la crise économique du début des années $1920^{7}$ et de l'inimité des milieux d'affaires japonais envers l'Indochine française dont le marché leur est fermé (p. 214-215).

On y apprend aussi au passage qu'il exista à Yokohama une "Maison commune de France $^{8} »($ p. 304) inaugurée le 23 décembre 1923 - trois mois à peine après le grand tremblement de terre qui toucha la région du Kantō -, qui fut construite grâce à la générosité de l'Indochine française et de la concession française de Shanghai pour venir en aide aux Français sinistrés de Yokohama.

15 Le projet de la Maison franco-japonaise se précise dans une lettre du 28 avril 1924 (p. 346-347) adressée à Raymond Poincaré, président du Conseil et ministre des Affaires étrangères : la Maison « devrait servir d'abri et de centre d'action aux Français d'élite 
qui viendraient y donner des conférences et des leçons. Les pensionnaires français suivraient pendant deux ou trois ans des cours de langue et d'écriture japonaises et étudieraient l'histoire, l'économie politique, l'art, la littérature du pays ${ }^{9} \%$. Claudel envisage donc cette Maison comme une «École des langues extrême-orientales [...] efficace et pratique pour former des jeunes gens qui sauraient parler et lire non seulement le japonais mais le chinois »(p. 346-347).

La création concrète de la Maison se réalise grâce à Murai Kichibei ${ }^{10}$, industriel du tabac et mécène francophile, qui met à disposition une villa de style européen située dans le quartier central de Kōjimachi à Tokyo (sur le terrain de l'actuel lycée Hibiya) (photo $\mathrm{n}^{\circ}$ 9, p. 376). Les conférenciers que Claudel envisage d'inviter sont des personnalités de premier plan du monde intellectuel, scientifique et artistique: Henri Bergson, Marie Curie, le sculpteur Antoine Bourdelle et Henri Focillon (p. 387). Claudel veut faire en effet de la Maison un «centre de la vie sociale et intellectuelle fréquenté par les Japonais et les étrangers » (p. 466).

17 La cérémonie d'inauguration a finalement lieu le 14 décembre 1924 au Club industriel du Japon à Marunouchi, en présence notamment du Premier ministre japonais et des ministres japonais des Affaires étrangères et de l'Instruction publique (p. 427). Une lettre du 26 juillet 1926 adressée à l'administrateur délégué de la Maison, Kijima Kōzō (ancien consul du Japon à Lyon), révèle la position de Claudel quant au partage des responsabilités entre la direction française qui «organise l'activité académique et contrôle les pensionnaires » et la partie japonaise qui «contrôle les finances » de la Maison (p. 505-506).

Claudel, infatigable, envisagea ensuite la création d'une maison semblable à Kyoto (25 avril 1926, p. 466) ou qui pourrait en être une "succursale d'été » (p. 485). Le plan en est précisé dans une correspondance à Aristide Briand (14 octobre 1926, p. 539-540) ${ }^{11}$. Il s'agirait d'une sorte d'« université d'été » française installée au Mont Hiei, haut lieu du bouddhisme qui avait séduit le géographe Francis Ruellan, l'un des deux premiers pensionnaires à la Maison franco-japonaise. Elle prévoyait, sur une période de trois ans, des études de la langue, de l'histoire, de la géographie et de la philosophie françaises, en vue de «former une élite de jeunes gens dont les plus brillants seront envoyés » à la Maison du Japon à la Cité universitaire internationale à Paris. On sait que ce projet donna finalement naissance en octobre 1927 à l'«Institut franco-japonais de Kyoto ", installé sur la colline de Kujōyama, et dont Ruellan fut le premier directeur jusqu'en 1931 (p. 577-578) ${ }^{12}$. Cette seconde institution fut créée, rappelons-le, exclusivement grâce à une souscription japonaise. Claudel, nommé ambassadeur à Washington, quitta le Japon le 17 février 1927 - peu après avoir assisté aux funérailles de l'empereur Taishō -, et n'en vit pas la réalisation concrète.

On comprend mieux à la lecture de cette précieuse chronologie comment Claudel a voulu - et réussi à faire - de la Maison franco-japonaise un outil de la diplomatie culturelle française au Japon, mais aussi un instrument pour l'étude approfondie du Japon par les Français, seul gage d'une véritable entente mutuelle.

Les 5 et 6 octobre 2013 se tiendra à la Maison franco-japonaise un colloque sur « Le rapprochement franco-japonais dans l'entre-deux-guerres » et il n'y a pas de doute que cette chronologie sera d'une grande utilité pour situer le contexte de ces échanges dans les années 1920 et le rôle déterminant de Claudel dans leur renforcement. 


\section{NOTES}

1. Chūjō Shinobu 中佟忍, Ōde Atsushi 大出敦, Shinonaga Nobutaka 篠永宣孝, Negishi Tetsurō 根 岸徹郎 (dir.), Nihon ni okeru Pōru Kurōderu. Kurōderu no tainichi nenpu 日本におけるポール・ク ローデルークローデルの帯日年譜Claudel au Japon 1898 ・1921-1927, Tokyo, Kuresu shuppan クレ ス出版, 2010.

2. Chūjō Shinobu 中條忍, Hasekura Takaharu 支倉崇晴 (dir.), Kurōderu to Nihon. Kurōderu botsugo gojū nen kokusai kaigi, shinpojiumu ronbun-shū クローデルと日本一クローデル歼後50年国際会 議・シンポジウム論文集 Claudel et le Japon: Cinquantenaire de la mort de Claudel. Actes du Colloque international et de la table ronde, Tokyo, Shichigatsudō 七月堂, 2006.

3. Ōde Atsushi 大出敦, Chūjō Shinobu 中佟忍, Negishi Tetsurō 根岸徹郎 (dir.), Nihon ni okeru Kurōderu 日本におけるクローデル Claudel au Japon, Tokyo, Kurōderu botsugo gojūnen kinen kikaku iinkai クローデル歿後50年記念企画委員会, 2005.

4. On complétera utilement ces informations par la lecture de l'histoire très documentée de la Maison franco-japonaise qui fut compilée par Bernard Frank et Iyanaga Shōkichi dans le $n^{\circ} 31$ de la revue Nichifutsu bunka 日仏文化 Revue de collaboration culturelle franco-japonaise ("numéro spécial consacré au cinquantenaire de la Maison franco-japonaise »), fasc. II, juillet 1974. On lira également les deux importants passages consacrés à la Maison franco-japonaise dans l'excellent ouvrage de Michel Wasserman, D'or et de Neige. Paul Claudel et le Japon, Paris, Gallimard, «Les Cahiers de la NRF », 2008, p. 100-110 et p. 160-168.

5. Voir sur ce point l'article de Chūjō Shinobu, Ebisu, n 26, 2001, p. 11-12.

6. Comme le précise une note p. 175, la subvention votée par le parlement japonais en 1923 sera finalement de 30000 yens et la souscription s'élèvera à 55000 yens en 1924. En 1927, Claudel indique qua la subvention annuelle de la MFJ accordée par le gouvernement français était de 42000 yens et celle de l'Indochine de près de 10000 yens. À titre indicatif, en 1927, la parité yen/ franc était d'environ de 1 pour 12.

7. Voir à ce propos «La crise économique au Japon. 17 mai 1922 », in Paul Claudel, Correspondance diplomatique. Tokyo, 1921-1927, textes choisis, présentés et annotés par Lucile Garbagnati, avec le concours du Centre Jacques-Petit, "Cahiers Paul Claudel », vol. 14, Paris, Gallimard, 1995, p. 136-139.

8. Elle est appelée diversement « Maison commune » ou « Maison de France de Yokohama » dans un texte de Claudel qui annonce son inauguration: "Les œuvres françaises de Tokyo et de Yokohama. 29 décembre 1923 », ibid., Paul Claudel, 1995, p. 235.

9. On trouvera le texte original sous le titre «La Maison franco-japonaise. 28 avril 1924 » dans ibid., Paul Claudel, 1995, p. 258-262.

10. Voir son portrait dans «Un ami de la France : M. Murai Kichibei », Japon et Extrême-Orient, $\mathrm{n}^{\circ}$ 11-12, novembre-décembre 1924, p. 393-394.

11. Voir le texte original sous le titre «Création d'une université d'été française sur le Mont Hiei à Kyoto. 14 octobre 1926 » dans op. cit., Paul Claudel, 1995, p. 382-384.

12. Voir «L'Institut franco-japonais de Kyoto. 10 janvier 1927 » dans op. cit., Paul Claudel, 1995, p. 392-399. 


\section{AUTEURS}

\section{CHRISTOPHE MARQUET}

Maison franco-japonaise,

UMIFRE 19 MAEE-CNRS 